\title{
Consecutive Monitoring of Interleukin-6 Is Needed for COVID-19 Patients
}

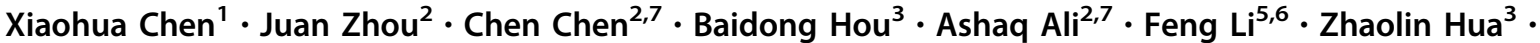 \\ Yingtao $\mathrm{Wu}^{1} \cdot$ Qin $\mathrm{Yang}^{1} \cdot$ Min Chen $^{1} \cdot$ Rong Zhang $^{4} \cdot$ Qianchuan Huang $^{1}$ (I) - Jinya Ding ${ }^{1}$ (I) \\ Xian-En Zhang ${ }^{3,7}$ (D) Dong Men $^{2,7}$
}

Received: 26 January 2021 / Accepted: 21 May 2021 / Published online: 7 July 2021

(c) Wuhan Institute of Virology, CAS 2021

Dear Editor,

The world is facing an immense challenge in the fight against coronavirus disease 2019 (COVID-19), which is caused by severe acute respiratory syndrome coronavirus 2 (SARS-CoV-2) (Gorbalenya et al. 2020). To date, tens of millions of people worldwide have been infected with SARS-CoV-2, and more than a million have died, and the situation continues. (Huang et al. 2021; Li et al. 2021) A retrospective study in the nick of time is necessary to renew knowledge against the disease. Cytokine release syndrome (CRS) features a dramatic increase of various pro-inflammatory cytokines and has been highlighted in COVID-19 patients; CRS is thought to be responsible for severe illness and high mortality in this disease (Moore and June 2020;

Xiaohua Chen, Juan Zhou and Chen Chen have contributed equally to this work.

Supplementary Information The online version contains supplementary material available at https://doi.org/10.1007/s12250021-00425-4.

Dong Men

d.men@wh.iov.cn

Xian-En Zhang

zhangxe@ibp.ac.cn

Jinya Ding

whzyydjy@163.com

$\triangle$ Qianchuan Huang

hqcwuhan@126.com

1 Department of Laboratory Medicine, General Hospital of Central Theater Command, PLA, Wuhan 430070, China

2 State Key Laboratory of Virology, Wuhan Institute of Virology, Center for Biosafety Mega-Science, Chinese Academy of Sciences, Wuhan 430071, China

3 National Laboratory of Biomacromolecules, CAS Center for Excellence in Biomacromolecules, Institute of Biophysics, Chinese Academy of Sciences, Beijing 100101, China
Ruan et al.2020). Therefore, cytokine concentrations may be useful for predicting the clinical outcomes of COVID-19, and anti-inflammatory strategies intended to regulate the cytokine pathway in COVID-19 patients are considered to be an alternative therapeutic route (Fu et al. 2020; Guan et al. 2020; Mehta et al. 2020).

Among CRS-related cytokines, interleukin-6 (IL-6), as a key mediator, has been a particular focus in COVID-19 infection (Aziz et al. 2020). IL-6 is a known pleiotropic cytokine with complex biological functions (VelazquezSalinas et al. 2019). It plays an essential role in activating and/or regulating various cell populations upon infections. However, the overexpression of IL-6 might promote disease progression. Several studies have suggested that the level of IL-6 is closely associated with COVID-19 progression because elevated IL-6 can be observed in severe and critically ill patients (Aziz et al. 2020; Chen et al. 2020b). In summary, elevated IL-6 has been identified as a predictor of COVID-19 severity (Han et al. 2020; Liu et al. 2020a; Ulhaq and Soraya 2020; Zhu et al. 2020), mechanical ventilation (Herold et al. 2020), and mortality risk (Laguna-Goya et al. 2020; Ruan et al. 2020; Zhou et al. 2020). Over the past few months, significant efforts have been made to clarify the relevance and roles of IL-6 in COVID-19. However, a robust analysis based on a large sample size has not yet been conducted. Moreover, most previous studies have been conducted based on single measurements of IL-6, and few studies have explored

4 Department of Laboratory Medicine, General Hospital of Southern Theater Command, PLA, Guangzhou 510000, China

5 State Key Laboratory of Virology, School of Basic Medical Sciences, Wuhan University, Wuhan 430071, China

6 Hubei Provincial Key Laboratory of Allergy and Immunology, Wuhan 430071, China

7 University of Chinese Academy of Sciences, Beijing 100039, China 
dynamic changes of IL-6 concentrations in COVID-19 patients (Han et al. 2020; Liu et al. 2020b).

In this retrospective study, a total of 1,453 confirmed COVID-19 patients who were admitted to Huoshenshan Hospital from 29 February to 14 March 2020 were enrolled, including 962 (66\%) moderate cases, 462 (32\%) severe cases, and $29(2 \%)$ critical cases. The demographics and clinical characterizations of the patients were listed in Supplementary Table S1. In this COVID-19 patient cohort, age was significantly associated with disease severity. There was no gender disparity; however, compared with women, more men had severe $(53 \%)$ or critical $(72 \%)$ illness. A relatively high percentage of patients had hypertension $(23 \% ; P<0.001)$ or diabetes $(12 \% ; P<0.001)$. In this cohort, hypertension and diabetes were significant risk factors for COVID-19.

Serum IL-6 concentrations in all patients were quantitatively determined using the Elecsys IL-6 detection kit (Roche Ltd). Concentrations of IL-6 in assorted groups were statistically analyzed and presented as the median (interquartile range; IQR). The median IL-6 concentrations for the moderate, severe and critical groups were $1.50 \mathrm{pg} / \mathrm{mL}$ $(1.50-3.65 \mathrm{pg} / \mathrm{mL}), 2.60 \mathrm{pg} / \mathrm{mL}(1.50-9.03 \mathrm{pg} / \mathrm{mL})$ and $60.92 \mathrm{pg} / \mathrm{mL} \quad(10.49-408.80 \mathrm{pg} / \mathrm{mL})$, respectively. Remarkably, the critical patients had significantly higher IL-6 concentrations compared with the moderate and severe patients $(P<0.001)$. Overall, $129(13.4 \%)$ of the moderate patients, $125(27.1 \%)$ of the severe patients, and $25(86.2 \%)$ of the critical patients had IL-6 values that were greater than the reference concentration $(7 \mathrm{pg} / \mathrm{mL})$. These results indicate that elevated IL-6 is associated with COVID-19 severity (Han et al. 2020) and this biomarker might be a predictor of critical illness.

Furthermore, consecutive IL-6 concentrations were recorded for 184 patients (729 total tests) over approximately one month during hospitalization. These patients included 62 moderate patients, 111 severe patients, and 11 critical patients. Among them, 161 patients were cured, 11 patients were deceased, and 12 patients were discharged. None of these 184 patients received tocilizumab therapy. The dynamic changes in IL-6 corresponded to the COVID-19 severity and clinical outcomes of the patients, as shown in Fig. 1. In the moderate group, 58 patients $(93.5 \%)$ were cured, 3 patients $(4.8 \%)$ were discharged and one patient (1.6\%) died. The total median IL-6 concentration of the moderate group was within the reference range throughout the monitoring period, while the one deceased patient demonstrated a remarkable increase in IL- 6 from 43.44 to $5000 \mathrm{pg} / \mathrm{mL}$ (Fig. 1A). In the severe group, 100 patients $(90.1 \%)$ were cured, 9 patients $(8.1 \%)$ were discharged and two patients $(1.8 \%)$ died. Similar to the moderate group, the median IL- 6 concentrations slightly fluctuated near the reference concentration over time. The two deceased patients had high IL- 6 concentrations that were above the reference value. It should be noted that the one patient who showed significant fluctuation in IL-6 that increased first and then decreased was cured at last (Fig. 1B). In the critically ill groups, 8 patients $(72.7 \%)$ who had significantly increased IL- 6 concentrations died, and 3 patients (27.3\%) who showed no significant changes in IL-6 finally were cured (Fig. 1C). Based on the above results, we believe that the IL- 6 concentration is closely related to disease progression, and in particular, dynamic monitoring of changes in IL-6 serum concentration may be helpful in predicting the development of COVID-19 regardless of clinical classification.

The dynamic changes in IL-6 concentrations corresponding to the clinical outcomes were further evaluated. All 11 deceased patients had high IL-6 concentrations, reaching up to $5,000 \mathrm{pg} / \mathrm{mL}$ in $54.5 \%$ of these cases (Fig. 1D). This finding indicates that a sharply elevated IL6 concentration may be an important warning index of mortality (Chen et al. 2020a; Grifoni et al. 2020; Ruan et al. 2020; Zhou et al. 2020). The IL-6 concentrations of the 161 cured patients ( 655 total tests) and the 11 deceased patients (44 total tests) were statistically analyzed (Fig. 1E). The median IL-6 concentrations of the cured and deceased groups were $3.43 \mathrm{pg} / \mathrm{mL}(1.50-26.44 \mathrm{pg} / \mathrm{mL})$ and $534.10 \mathrm{pg} / \mathrm{mL}(64.20-1,815.50 \mathrm{pg} / \mathrm{mL})$, respectively. The deceased group clearly showed a higher mean IL- 6 concentration than the cured group. In addition, receiver operating characteristic curve (ROC) was used to evaluate the ability of IL-6 concentration to predict COVID-19 outcomes (Fig. 1F). The area under the curve was 0.93 (95\% CI $=0.90-0.96)$, and the optimal IL-6 cutoff value was $31.57 \mathrm{pg} / \mathrm{mL}$, with a sensitivity of $93.2 \%$ and a specificity of $76.3 \%$ according to the maximum Youden index. In our cohort, IL-6 concentrations greater than $31.57 \mathrm{pg} / \mathrm{mL}$ were associated with elevated mortality risk, with a positive predictive value of 0.21 and a negative predictive value of 0.99 . The above results further suggest the availability of IL-6 to predict the prognosis of COVID-19.

In conclusion, serum IL-6 concentrations in COVID-19 patients were evaluated in assorted groups. Overall, 13.4\% of moderate patients, $27.1 \%$ of severe patients, and $86.2 \%$ of critical patients had elevated IL-6 concentrations, suggesting a correlation between IL- 6 and disease severity. Notably, the dynamic changes in IL-6 concentration corresponded to COVID-19 severity and clinical outcomes. The majority of the moderate and severe patients showed no significant changes in IL- 6 levels over time, and the final cure rates in these patient groups were $93.5 \%$ and $90.1 \%$, respectively. In contrast, the critically ill patients showed obviously increased IL- 6 concentrations, which was associated with a high fatality rate of $72.7 \%$. Overall, the deceased patients demonstrated a fairly high IL-6 level 

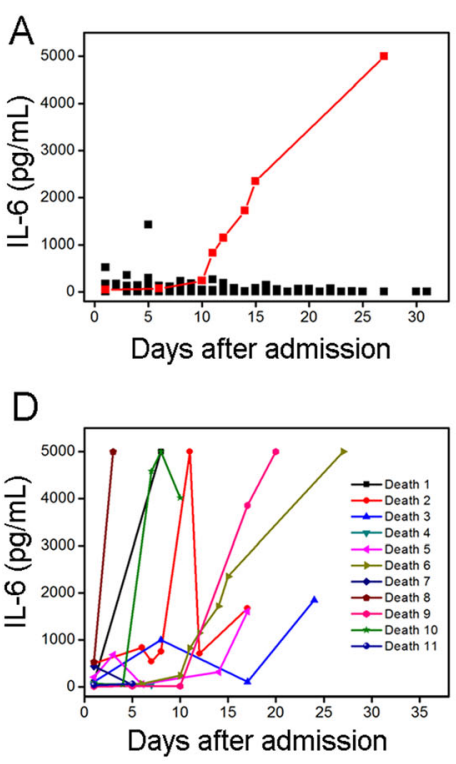
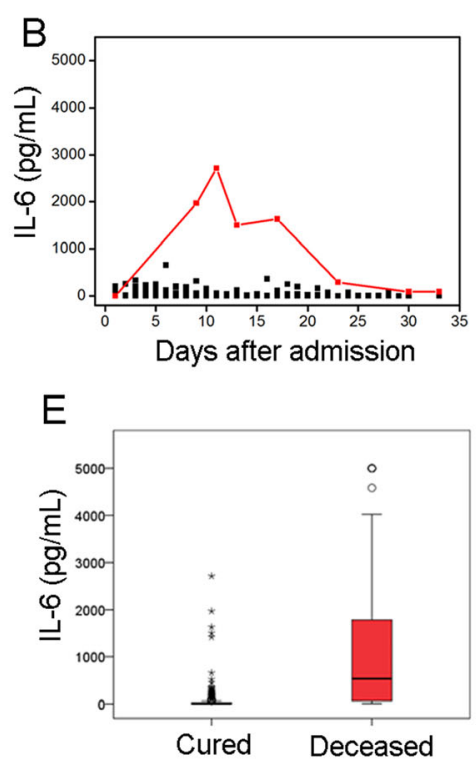
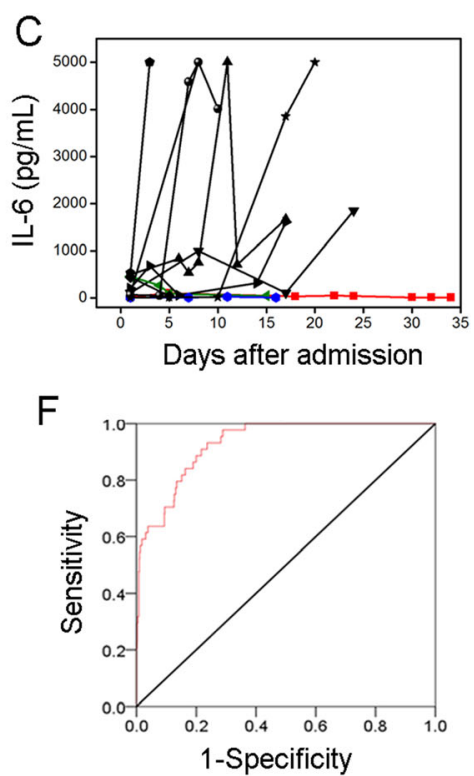

Fig. 1 Evaluation of IL-6 changes in 184 patients with confirmed COVID-19. A Dynamic changes in IL- 6 concentration of 62 moderate patients after admission. One deceased patient with a remarkable increase in IL-6 concentrations was highlighted in red. B Dynamic changes in IL-6 concentration of 111 severe patients after admission. One cured case with IL-6 increased first and then decreased was

compared with the cured patients. Although the relationship between elevated IL-6 and COVID-19 progression is not entirely clear, these findings suggest that changes in the IL- 6 concentration could be a good predictor of COVID-19 prognosis. We suggest that the dynamic changes in IL-6 should be monitored throughout the disease duration. This monitoring may have predictive value for changes in disease severity, as well as clinical value for applying timely and appropriate treatment.

Acknowledgements This work was partially supported by the National Key Research and Development Program of China (Grant No. 2020YFC0861100), the National Natural Science Foundation of China (Grant No. 82002744), the Strategic Priority Research Program of the Chinese Academy of Sciences (Grant No. XDB29050100), the Scientific Research Fund of Traditional Chinese Medicine of Hubei Health Commission (Grant No. ZY2021M035), the Youth Innovation Promotion Association of CAS (Grant No. 2014308) and the Interdisciplinary Innovation Team of CAS.

\section{Compliance with Ethical Standards}

Conflict of interest The authors declare that they have no conflict of interest.

Animal and Human Rights Statement Additional informed consent was obtained from all patients for which identifying information is included in this article. highlighted in red. C Dynamic changes in IL-6 concentration of 11 critically ill patients after admission. Three cured patients with slight change in IL-6 were highlighted in blue, green and red respectively. D Dynamic changes in IL-6 concentration of 11 deceased patients. E IL-6 levels in cured and deceased patient. F ROC curve of IL-6 for predicting COVID-19 mortality.

\section{References}

Aziz M, Fatima R, Assaly R (2020) Elevated interleukin-6 and severe COVID-19: a meta-analysis. J Med Virol 92:2283-2285

Chen T, Wu D, Chen H, Yan W, Yang D, Chen G, Ma K, Xu D, Yu H, Wang H, Wang T, Guo W, Chen J, Ding C, Zhang X, Huang J, Han M, Li S, Luo X, Zhao J, Ning Q (2020a) Clinical characteristics of 113 deceased patients with coronavirus disease 2019: retrospective study. BMJ-Brit Med J 368:m1295

Chen X, Zhao B, Qu Y, Chen Y, Xiong J, Feng Y, Men D, Huang Q, Liu Y, Yang B, Ding J, Li F (2020b) Detectable serum severe acute respiratory syndrome coronavirus 2 viral load (RNAemia) Is closely correlated with drastically elevated interleukin 6 level in critically ill patients with coronavirus disease 2019. Clin Infect Dis 71:1937-1942

Fu YJ, Cheng YX, Wu YT (2020) Understanding SARS-CoV-2mediated inflammatory responses: from mechanisms to potential therapeutic tools. Virol Sin 35:266-271

Gorbalenya AE, Baker SC, Baric RS, de Groot RJ, Drosten C, Gulyaeva AA, Haagmans BL, Lauber C, Leontovich AM, Neuman BW, Penzar D, Perlman S, Poon LLM, Samborskiy DV, Sidorov IA, Sola I, Ziebuhr J, Coronaviridae Study G (2020) The species severe acute respiratory syndrome-related coronavirus classifying 2019-nCoV and naming it SARS-CoV-2. Nat Microbiol 5:536-544

Grifoni E, Valoriani A, Cei F, Lamanna R, Gelli AMG, Ciambotti B, Vannucchi V, Moroni F, Pelagatti L, Tarquini R, Landini G, Vanni S, Masotti L (2020) Interleukin-6 as prognosticator in patients with COVID-19. J Infect 81:466-467

Guan WY, Lan WD, Zhang J, Zhao S, Ou JX, Wu XW, Yan YQ, Wu JG, Zhang QW (2020) COVID-19: antiviral agents, antibody development and traditional chinese medicine. Virol Sin 35:685-698

Han H, Ma Q, Li C, Liu R, Zhao L, Wang W, Zhang P, Liu X, Gao G, Liu F, Jiang Y, Cheng X, Zhu C, Xia Y (2020) Profiling serum 
cytokines in COVID-19 patients reveals IL-6 and IL-10 are disease severity predictors. Emerg Microbes Infect 9:1123-1130

Herold T, Jurinovic V, Arnreich C, Lipworth BJ, Hellmuth JC, von Bergwelt-Baildon M, Klein M, Weinberger T (2020) Elevated levels of IL-6 and CRP predict the need for mechanical ventilation in COVID-19. J Allergy Clin Immunol 146:128-136

Huang CL, Wang YM, Li X, Ren LL, Gu XY, Kang L, Guo L, Liu M, Zhou X, Luo JF, Huang ZH, Tu SJ, Zhao Y, Chen L, Xu DC, Li YP, Li CH, Peng L, Li Y, Xie WX, Cui D, Shang LH, Fan GH, Xu JY, Wang G, Wang Y, Zhong JC, Wang C, Wang JW, Zhang DY, Cao B (2021) 6-month consequences of COVID-19 in patients discharged from hospital: a cohort study. Lancet 397:220-232

Laguna-Goya R, Utrero-Rico A, Talayero P, Lasa-Lazaro M, Ramirez-Fernandez A, Naranjo L, Segura-Tudela A, CabreraMarante O, Rodriguez de Frias E, Garcia-Garcia R, FernandezRuiz M, Maria Aguado J, Martinez-Lopez J, Ana Lopez E, Catalan M, Serrano A, Paz-Artal E (2020) IL-6-based mortality risk model for hospitalized patients with COVID-19. J Allergy Clin Immunol 146:799-807

Li TB, Huang T, Guo C, Wang AL, Shi XL, Mo XF, Lu QQ, Sun J, Hui TT, Tian G, Wang LY, Yang JL (2021) Genomic variation, origin tracing, and vaccine development of SARS-CoV-2L: a systematic review. The Innov 2:100116

Liu F, Li L, Xu M, Wu J, Luo D, Zhu Y, Li B, Song X, Zhou X (2020a) Prognostic value of interleukin-6, C -reactive protein, patients with COVID-19 and procalcitonin in patients with COVID-19. J Clin Virol 127:104370
Liu T, Zhang J, Yang Y, Ma H, Li Z, Zhang J, Cheng J, Zhang X, Zhao Y, Xia Z, Zhang L, Wu G, Yi J (2020b) The role of interleukin-6 in monitoring severe case of coronavirus disease 2019. EMBO Mol Med 12:e12421

Mehta P, McAuley DF, Brown M, Sanchez E, Tattersall RS, Manson JJ, Speciality HLHA (2020) COVID-19: consider cytokine storm syndromes and immunosuppression. Lancet 395:1033-1034

Moore JB, June CH (2020) Cytokine release syndrome in severe COVID-19. Science 368:473-474

Ruan Q, Yang K, Wang W, Jiang L, Song J (2020) Clinical predictors of mortality due to COVID-19 based on an analysis of data of 150 patients from Wuhan, China. Intensive Care Med $46: 846-848$

Ulhaq ZS, Soraya GV (2020) Interleukin-6 as a potential biomarker of COVID-19 progression. Med Mal Infect 50:382-383

Velazquez-Salinas L, Verdugo-Rodriguez A, Rodriguez LL, Borca MV (2019) The role of interleukin 6 during viral infections. Front Microbiol 10:1057

Zhou F, Yu T, Du R, Fan G, Liu Y, Liu Z, Xiang J, Wang Y, Song B, Gu X, Guan L, Wei Y, Li H, Wu X, Xu J, Tu S, Zhang Y, Chen H, Cao B (2020) Clinical course and risk factors for mortality of adult inpatients with COVID-19 in Wuhan, China: a retrospective cohort study. Lancet 395:1054-1062

Zhu Z, Cai T, Fan L, Lou K, Hua X, Huang Z, Gao G (2020) Clinical value of immune-inflammatory parameters to assess the severity of coronavirus disease 2019. Int J Infect Dis 95:332-339 